\title{
Distributed Resource Allocation in Ad Hoc Networks
}

\author{
Zhijun Cai and Mi Lu \\ Department of Electrical Engineering \\ Texas A\&M University, College Station, TX 77843
}

\begin{abstract}
An ad-hoc network is a collection of wireless mobile nodes without the required intervention of any centralized Access Point. With the existence of the hidden terminals and the absence of the central control, the resource allocation is always a very challenging problem in such networks. In this paper, a distributed resource allocation scheme is designed for ad hoc networks, in which each mobile node dynamically searches its Native Index (NI) with negligible communication overhead. By utilizing the NI, the hidden terminal problem can be completely avoided in the resource allocation, and the resource can be efficiently distributed to all the nodes. The proposed resource allocation method can greatly improve the system throughput. Utilizing the method, the broadcast problem can be easily solved and QoS service (Quality of Service) can be efficiently supported.
\end{abstract}

\section{Introduction and Backgrounds}

An ad-hoc network is a collection of wireless mobile nodes which has no centralized control unit. Every node has limited transmission range, hence the packets may be relayed over multiple nodes before the destination node is reached. It is widely used in many cases, such as battlefields, search-and-rescue operations, disaster environments. Designing an efficient resource allocation scheme for such networks is always a very challenging problem due to the following reasons.

1. Hidden and exposed terminal problems. Such problems are caused mainly due to the limited transmission radius of the mobile nodes or the unsatisfied wireless environments. If one node is in the range of the receiver, but not the transmitter, the node is termed a hidden terminal to the transmitter. Similarly, an exposed terminal is a node which is in the range of the transmitter but not the receiver.

2. When nodes communicate, two types of collisions may arise [1]. The first type of collisions, termed the primary collision, means that a node transmitting a packet, cannot be receiving a packet at the same time and this also implies the converse. The second type of collision, termed the secondary collision, means that a node can not receive more than one packets at the same time. In both cases, all packets are rendered useless. 
Much work has been done on the resource allocation in ad hoc networks [2-7]. In [2], different kinds of code assignment schemes have been discussed. Two phase algorithms have been devised to assign the codes to the transmitters, receivers and to pair of stations. In [3], an adaptive scheme has been proposed to allocate a portion of the available bandwidth only to those users who are active. The scheme places the nodes in different groups and dynamically divides the total network capacity between the groups. The communication overhead of the existing resource assignment methods is high due to the dynamic network topology, which increases the system offered load. In this paper, a distributed resource allocation scheme is designed for ad hoc networks, in which each mobile node dynamically searches its Native Index (NI) with negligible communication overhead. By utilizing the NI, the hidden terminal problem can be completely avoided in the resource allocation, and the resource can be efficiently distributed to all the nodes.

The rest of this paper is organized as follows. The proposed resource allocation scheme is illustrated in Section 2. Section 3 describes the solution to the broadcast problem and the support of the QoS service. Section 4 gives the performance analysis and the simulation results, and we conclude in Section 5 .

\section{Proposed Resource Allocation Scheme}

\subsection{Network Model}

In an ad hoc network consisting of $N$ nodes, the packets may be relayed over multiple nodes before the destination node is reached. An example is shown in Figure 1. An identical transmission radius is assumed for all nodes. Each node is given a unique identification number (ID). For a given node $A$, if the shortest number of hops between $A$ and a particular node is $\leq h$, then that node is an $h$-hop neighbor of $A$. Denote $\delta_{h}(A)$ as the set of all $h$-hop neighbors of $A$. For example, in Figure $1, \delta_{1}(A)=\{M, B, C\}$. In the following part, the meaning of "neighbors" is identified as 1-hop neighbors. Define $\delta_{h}$ as the maximum size of $\delta_{h}(X)$ (for all $X ; X$ is a random node in the network). We assume $\delta_{2}$ is upper-bounded by $D$.

\subsection{NI Distribution}

In the proposed resource allocation scheme, each node should obtain its NI (NI $\in\{1,2,3, \cdots, D, D+1\}$; the set of $\{1,2,3, \cdots, D, D+1\}$ is termed the NI set), and the NI distribution should satisfy the condition that any two nodes within 2-hop distance should not have the same NI. The NI for the node will be updated when the network topology changes.

In ad hoc networks, each node should identify its neighbors after poweron (known as the neighbor-detection problem), and the general solution to the problem is the periodical beacon signal broadcast, in which each node broadcasts its beacon signal periodically to identify itself after power-on. We next propose a 


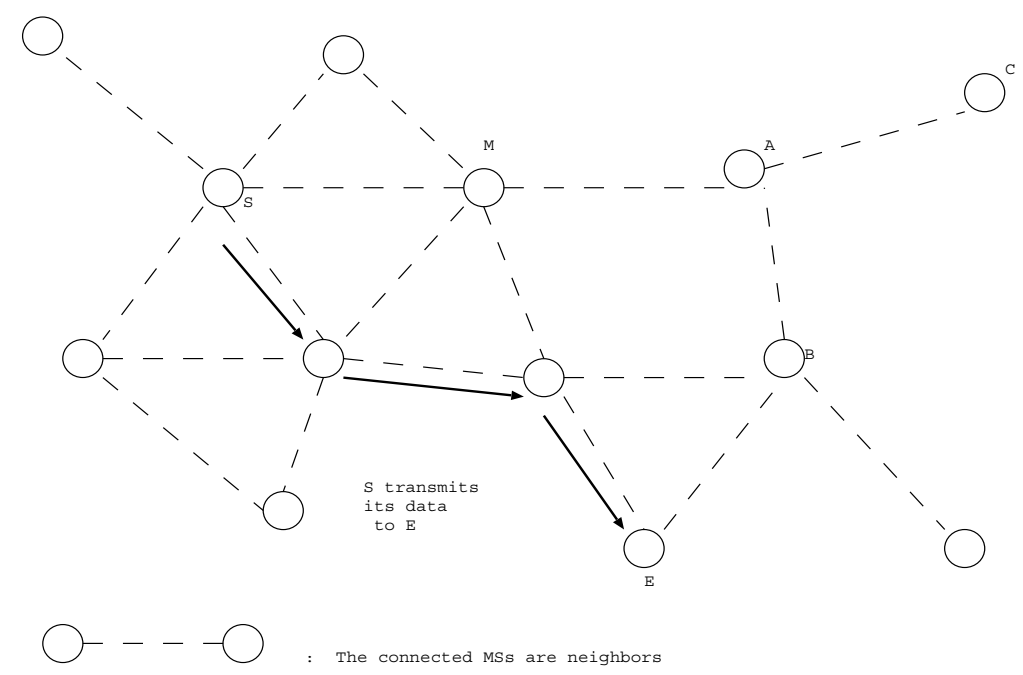

Fig. 1. A multihop network.

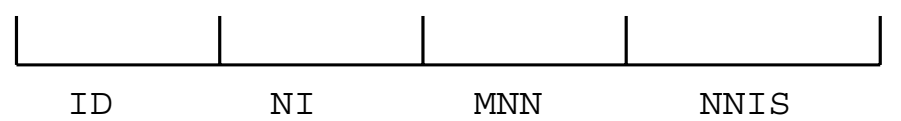

Fig. 2. Format of the beacon signal.

method to jointly solve the NI distribution problem with the neighbor-detection problem. The format of the beacon signal is as Figure 2. For the beacon signal of $A$, the $I D$ filed and the $N I$ field are $A$ 's ID and $A$ 's NI respectively. Initially $A$ will set its NI to NULL after power-on. The $M N N$ field is the minimum node ID of $A$ 's neighbors whose NI is still NULL, while the NNIS field is the set of the NIs which have been utilized by $A$ 's neighbors. Through the periodical broadcast of the beacon signal, every node will be aware of the NIs, the MNNs and the NNISs of its neighbors. For a given node $A$, its Available NI Set (ANIS) includes all the remaining NIs that are not occupied by the nodes in $\delta_{2}(A)$, which is the remaining set subtracted from the NI set by all NNISs of $A$ 's neighbors. Every node should maintain its up-to-date ANIS. The NI obtaining process is as follows. If the NI of $A$ is NULL and $A$ acknowledges that its ID is the minimum ID of its 2-hop neighbors whose NIs are still NULL, which means all MNNs of its neighbors are equal to $A$ 's ID, $A$ will set its NI to a randomly selected NI from its ANIS. We have the following results.

1. The ANIS of a node will not be empty when the node attempts to obtain its NI, since the number of NIs $(D+1)$ is greater than the maximum number of 2 -hop neighbors for any node plus 1. 
2. Any two nodes within 2-hop distance can not set their NIs at the same time, since only when the node acknowledges that its ID is the minimum ID of all its 2-hop neighbors whose NIs are still NULL, it can set its NI. Therefore, the NIs can be distributed without any conflict (the conflict means the case that two nodes within 2-hop distance set their NIs to the same one due to the unawareness of each other).

3. Due to the dynamic topology, if one link is broken, no nodes need to update its NI, while if one link is generated, at most two nodes may need to update their NIs. The reason is as follows. Suppose all nodes have already obtained their NIs. If one link is broken, the NI distribution condition can still be satisfied. Hence no NI update is required. If one link is generated, suppose the link between $A$ and $B$ is generated. Then $A$ will become a new 2-hop neighbor of $B$ 's neighbors. If $A$ and any neighbor of $B$ have the same NI, the node with the smaller ID should set its NI to NULL, and will initiate the NI obtaining process. So does node $B$. Therefore, at most two nodes are required to update their NIs. The property shows the stability of the proposed NI distribution method. If a new node is added into the network, the node will set its NI to be NULL, and initiate the NI obtaining process.

\subsection{Resource Distribution}

Two types of collisions may occur during the data communications in ad hoc networks as we have discussed in Section 1. The resource should be assigned to the nodes to avoid the potential collisions.

The resource in the ad hoc network can be represented by the codes, the slots or the frequency channels which corresponds to the CDMA (Code-Division Multiple Access), TDMA (Time-Division Multiple Access) or FDMA (FrequencyDivision Multiple Access) respectively. However, only in the TDMA, two types of collisions are possible to be completely avoided. The reason is as follows. Suppose the resource is represented by the codes, and each node obtain an orthogonal code to transmit its data. The code distribution satisfies the condition that any two nodes within 2-hop distance should not have the same orthogonal code. Utilizing the NI, the code distribution can be simply realized (each NI is an index to an orthogonal code; each node should utilize the code corresponding to its NI). Hence, the secondary collision can be completely avoided. However, the primary collisions still exist since the transmission of the nodes is not scheduled. For example, in the network topology shown in Figure 3, $A$ and $B$ are neighbors while $B$ and $C$ are neighbors. If $A$ and $C$ transmit the data to $B$ simultaneously, no collisions will occur due to the orthogonal codes. However, if $A$ transmits the data to $B$ while $B$ transmits the data to $C, B$ will not obtain the data from $A$ although the codes have been perfectly distributed. Therefore, if the resource has been represented by the codes, the transmission of the nodes should be scheduled to avoid the primary collisions, which is not an easy task to realize in the distributed environments such as ad hoc networks. Similarly, if the resource is represented by the frequency channels, the primary collision can not be avoided either. 


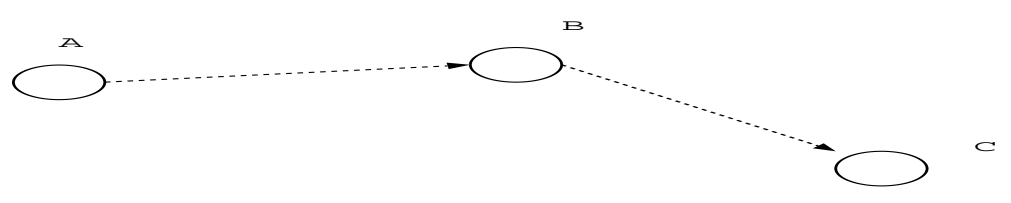

Fig. 3. An example of the resource distribution.

If the resource has been represented by the slots, the secondary collision can be avoided as well as the primary collision. The slot distribution is as follows. The frame is composed of $D+1$ slots. Each NI is an index to a unique slot, and each node should utilize the slot corresponding to its NI to transmit its data. The primary collision will not exist since any two neighbor nodes do not transmit the data in the same data slot. Moreover, the secondary collision will be completely avoided due to the slot distribution condition. In the proposed resource allocation scheme, the network resource is represented by the time slots.

\subsection{Dynamic NI Scheme}

Since NI is $\in\{1,2,3, \cdots, D+1\}$, if the estimated $D$ is smaller, then some nodes may not obtain its NI, which implies these nodes can not have their resource. To address the problem, the dynamic NI scheme can be adopted. If a node does not utilize its NI (which corresponds to its resource) for some time, the node should release its NI, and set its NI to 0 (not NULL). Then other nodes can utilize the released NI. When the node attempts to transmit the data again, the node should set its NI to NULL and initiate the NI obtaining process.

\section{The Broadcast Problem and the QoS Service}

\subsection{Solution to the Broadcast Problem}

The broadcast problem is one of the fundamental issues in ad hoc networks, and much work has been done $[1,9,10]$. The existing methods can be divided into two groups, topology-dependent scheme and topology independent scheme. In the topology dependent scheme, global or part of the network topology are utilized to determine the minimum broadcast frame length and transmission schedule, while in the topology independent scheme, the mathematics theories such as the Galois Field theory have been adopted to achieve the efficient solutions with the topology-independent property. The topology-dependent scheme can always produce better solution than the topology independent scheme since the topology information has been utilized to determine the transmission schedule. Hence, if the network topology is fixed or changes slowly, the topologydependent scheme is the choice. However, in the mobile networks, since the topology-dependent scheme entails significant control overhead to determine the 
schedule, the topology-independent scheme achieves its gain due to its transparency to the network topology. In our proposed solution, since the NI distribution is corporated into the neighbor detection process, the control overhead to distribute the NI is negligible. Then a new topology-dependent scheme with negligible communication overhead can be proposed. Suppose the time is divided into frames, and each frame is composed of $D+1$ data slots. The entire network is synchronized on the frame and the slot basis. For a given node, say $A$, suppose its NI is $k$. Then $A$ should utilize the $k t h$ slot to broadcast its data. No conflicts exist when a node broadcasts its data according to the NI distribution condition.

\subsection{Designing a MAC (Media Access Control) to Support the QoS}

Presently QoS service in ad hoc networks becomes more and more important. For the QoS service, the resource should be allocated on demand (not pre-allocated). The existing on-demand resource allocation methods in ad hoc networks are based on the REQ/ACK dialogue, which is not a collision-free scheme (the REQ and the ACK packets suffer collisions). When the service load of the network is high, due to the collisions, the existing methods perform pretty bad. Next we will propose an efficient MAC to support the QoS. In the proposed method, very little resource will be pre-allocated to every node to avoid the collisions among the resource control packets (the REQ packets and the ACK packets), while the major part of the resource is allocated on-demand.

Time is divided into frames, which is composed of the control subframe and the data subframe. The control subframe is composed of $D+1$ control slots while the data subframe is composed of $n_{2}$ data slots. The structure of the frame is illustrated in Figure 4. Each control slot has three fields, the Active Field (AF),

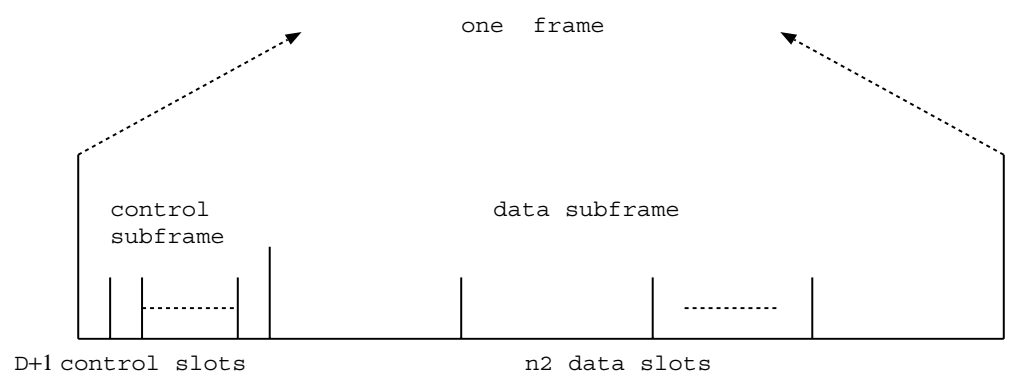

Fig. 4. Frame structure.

the Passive Field (PF) and the Allocation Acknowledgment Field (AAF). A node with its NI as $k$ should utilize the AF of the $k t h$ control slot to transmit its control packets. From the NI distribution condition, no collisions will occur for the control packets. For a given node, say $A$, its control packet should include the resource request information, $A$ 's neighbor set, etc. 


\section{Resource Status}

For every node, in each data slot, it can either transmit or receive the data. For a given node $A$, its Receiving Slot Set (RSS) includes all the data slots that $A$ utilizes to receive the data, and its Transmitting Slot Set (TSS) includes all the data slots that $A$ utilizes to transmit the data. Its Available Receiving Slot Set (ARSS) includes all the available data slots that $A$ can utilize to receive the data (but not occupied yet). Due to the hidden terminal problem, the ARSS of $A$ includes the slots in which none of $A$ 's neighbors including $A$ will transmit the data.

\section{Resource Reservation Process}

Suppose $A$ attempts to establish a QoS link to one of its neighbor $B$. $A$ will transmit a resource request to $B$ in the AF during its control slot. Every neighbor of $A$ will be aware of the request from $A$. Then all the neighbors of $A$ except $B$ will transmit its RSS while $B$ transmits its ARSS. The transmission strategy is as follows. Since $A$ includes all the IDs of its neighbors in the control packet, every neighbor of $A$ can obtain a sequence number according to the increasing order of all the IDs. For example, suppose $A$ has three neighbors, $B, C$ and $D$. Assume $I D(B) \leq I D(C) \leq I D(D)$. Then $B$ will obtain the sequence number, 1. The sequence number of $C$ is 2 and $D$ is 3 . The PF field is composed of $\delta_{1}$ mini-fields. Each neighbor of $A$ will transmit its RSS or ARSS in a certain minifield according to its sequence number. In the above example, $B$ will transmit its ARSS is the 1 st mini-field, while $C$ transmits its RSS in the $2 n d$ mini-field. $D$ will utilize $3 r d$ mini-field to transmit its RSS. Considering the definition of $\delta_{1}$, for a given node, it will receive all the RSSs and the ARSS from its neighbors safely. Based on the received information, $A$ will determine whether and how to allocate the resource for its request. The determination process is as follows. First, $A$ will calculate the available slot set. Suppose the received RSS sets are $R S S_{1}, R S S_{2}, R S S_{3}, \ldots, R S S_{k}$, and the received ARSS is $A R S S$. Then $A$ will calculate its available slot set as $\overline{\left(\bigcup_{i=1}^{i=k} R S S_{i}\right) \cup(T S S)} \cap(A R S S)$. Here, TSS means the TSS of $A$. Then it will check whether the available slots are enough for the QoS requirement. If so, it will allocate the required slots for the service. Otherwise it will determine whether to decrease the QoS requirements to utilize the available slots. After that, $A$ will transmit its allocation result in the AAF during the same control slot, and every neighbor of $A$ will receive the information in the AAF. Based on that, the destination node $B$ will update its ARSS and RSS while other neighbor nodes of $A$ update their ARSS. $A$ should also update its own RSS, TSS and the ARSS. Moreover, $B$ will acknowledge the data slots to receive the data from $A$. The proposed resource reservation process is requesterbased (the node which transmits the resource request is termed the requester). That is, if a node attempts to establish the QoS link to another node, it will transmit its request in the AF to query the current local resource information. During the PF, the node will be aware of the current local resource status, and the node can determine how to utilize the available resource to fulfill its QoS requirement. The requester has more flexibility to determine how to utilize the resource. After $A$ has reserved the data slots, it will transmit the data safely in 
the reserved data slots during every frame. If $A$ finishes its data transmission, $A$ should transmit a resource release message in its control slot, which will update the RSS and the ARSS of $A$ 's neighbors including $B$. $A$ should also update its own TSS, RSS and ARSS.

\section{Performance Analysis and Simulation Results}

\subsection{NI Distribution}

Since the NI distribution has been solved together with the neighbor detection problem, the communication overhead is negligible. Due to the dynamic topology, the NI should be updated. The following advantages can be obtained by adoption of the proposed scheme.

1. Local update property, which means the local topology change only affects the local nodes. This is very important to the large mobile networks.

2. Less control overhead. To realize the NI distribution, only the beacon signal is utilized, which is very short $(\approx 2 \log M+\log D+D$ bits $)$.

3. Dynamic NI scheme. If a node does not utilize its NI (which corresponds to its resource) for some time, the node should release its NI, and set its NI to 0 (not NULL).

4. Simplicity. The implementation of the NI distribution is simple since only minor changes have been made to the existing system.

\subsection{Network Broadcast}

Assume one node, say $A$, attempts to broadcast a packet to the network. Define the distance between node $X$ and node $Y$ as the minimum hop distance between $X$ and $Y$, termed $\operatorname{dis}(X, Y)$. Suppose $\max (\operatorname{dis}(A, X))=h(X$ is a random node in the network). Then the time cost of the broadcast will be $h \times$ Frame_Length, and $N$ broadcast packets will be transmitted. Note that each node will broadcast the packet once.

\subsection{QoS-Supported Resource Allocation}

In the proposed scheme, utilizing the $(D+1)$-slot control subframe, every node can transmit its control packet without any collisions, and the network resource can be adaptively allocated to the nodes upon their requests. Due to the collisionfree property among the control packets, the system throughput can steadily increased with the system overload. Next we use simulations to show the performance. The number of nodes in the simulated network is 100 , and $n_{2}=32$. Assume the network is degree-bounded network with $\delta_{1} \leq 8$, and $D=30$. Define the offered load as the average number of data packets arrived during one data slot for the network and the throughput as the number of data packets transmitted successfully during one data slot. The offered load is uniquely distributed to all nodes. The length of the control slot and the data slot is 64,2048 bytes 
respectively. Figure 5 illustrates the throughput performance. It is seen that the throughput is steadily increased with the system load. Note that the scheme also performs pretty good in light or medium load. Furthermore, we use simulation to

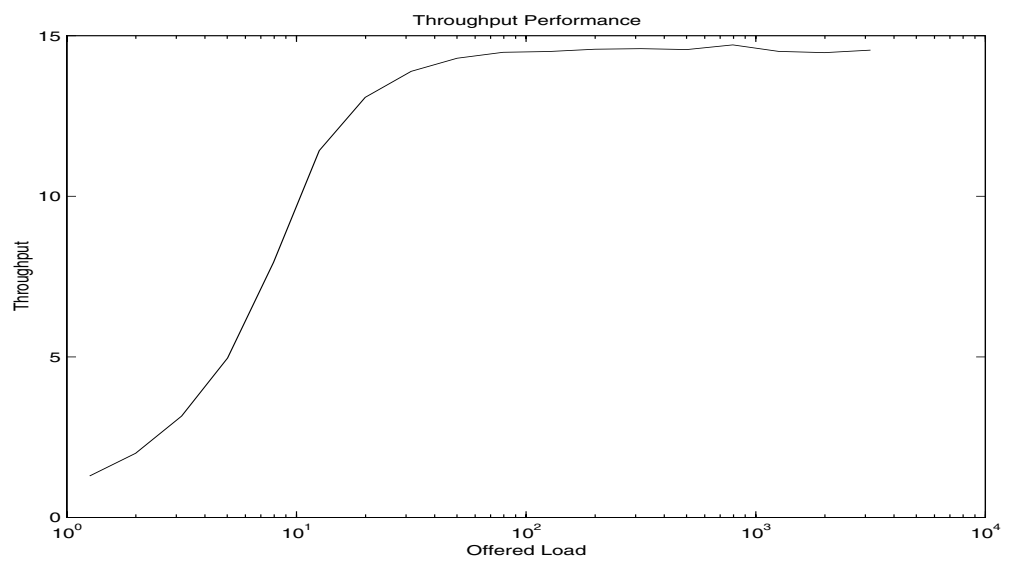

Fig. 5. Throughput performance.

show the network capacity for QoS requests. Assume $n$ QoS requests to reserve the resource arrive at the network, and is uniquely distributed to all the nodes. If the request can not be accepted (not enough resource), the request is failed. Assume all the requests attempt to reserve 2 slots. We illustrate the number of requests that can be accepted utilizing the proposed QoS-supported resource allocation scheme in Figure 6. It is seen that the proposed QoS-Supported scheme can accept most of the QoS requests simultaneously when $n \leq 220$, which represents the network capacity.

\section{Conclusions}

In this paper, a distributed resource allocation scheme is designed for ad hoc networks, in which each mobile node dynamically searches its NI with negligible communication overhead. By utilizing the NI, the hidden terminal problem can be completely avoided in the resource allocation, and the resource can be efficiently distributed to all the nodes. The proposed resource allocation method can greatly improve the system throughput. Utilizing the method, the broadcast problem can be easily solved and QoS service can be efficiently supported. The performance of the proposed scheme has been analyzed and verified by the simulation results. It is worth while to put continuous efforts in this and related research areas.

(This research was supported by the Texas Advanced Technology Program under Grant No. 000512-0039-1999.) 


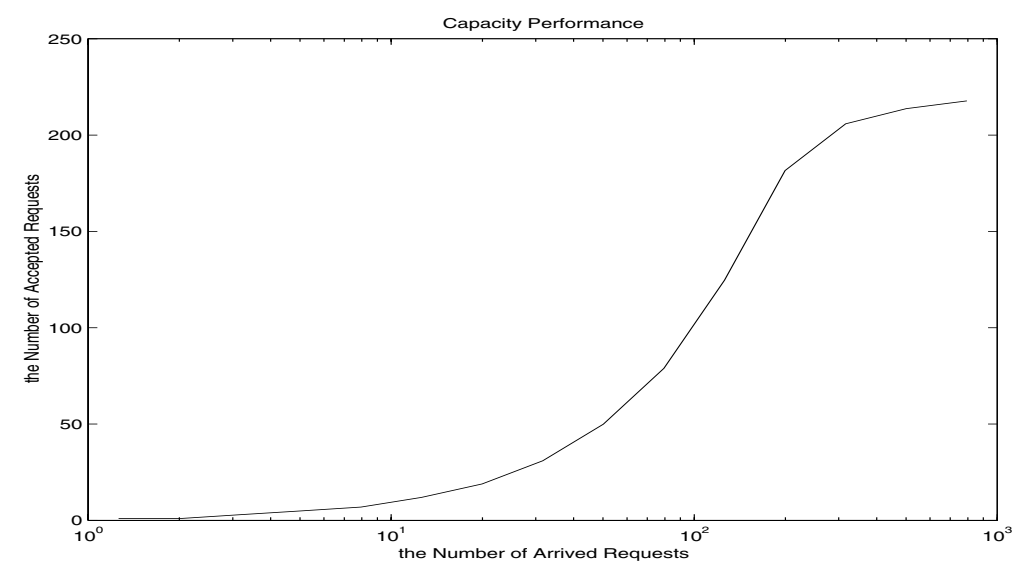

Fig. 6. Accepted QoS requests.

\section{References}

1. I. Chlamtac and A. Farago, "Making Transmission Schedules Immune to Topology Changes in Multi-hop Packet Radio Networks," IEEE Trans. Networking, vol. 2, no. 1, pp. 23-29, 1994.

2. Limin $\mathrm{Hu}$, "Distributed Code Assignments for CDMA Packet Radio Networks," IEEE Trans. Networking, vol. 1, no. 6, pp. 668-677, December 1993.

3. Y. Mohamed, M. K. Gurcan, and L. F. Turner, "A Novel Resource Allocation Scheme For Ad Hoc Radio Local Area Networks ," in Proc. of VTC'97, 1997, pp. 1301-1305.

4. Sanjay Lal and E. S. Sousa, "Distributed Resource Allocation For DS-CDMAbased Multimedia Ad Hoc Wireless LANs," IEEE Journal on Selected Areas in Communications, vol. 17, pp. 947-967, May 1999.

5. C. Trabelsi, "Access Protocol for Broadband Multimedia Centralized Wireless Local Area Network," in Proc. of the 2nd IEEE Symposium on Computers and Communications, 1997 , pp. 540-544.

6. A. M. Chou and V. O. K. Li, "Slot Allocation Strategies for TDMA protocols in Multihop Packet Radio Networks," in Proc. of IEEE INFOCOM'92, 1992, pp. 710-716.

7. R. Ramaswami and K. K. Parhi, "Distributed Scheduling of Broadcasts in a Radio Network," in Proc. of IEEE INFOCOM'89, 1989, pp. 497-504.

8. J. Whitehead, "Distributed Packet Dynamic Resource Allocation (DRA) for Wireless Networks," in Proc. of the 46th IEEE VTS, 1996, pp. 111-115.

9. Stefano Basagni, Danilo Bruschi, and Imrich Chlamtac, "A Mobility-Transparent Deterministic Broadcast Mechanism for Ad Hoc Networks," IEEE Trans. Networking, vol. 7, no. 6, pp. 799-807, Dec. 1999.

10. I. Chlamtac and O. Weinstein, "The Wave Expansion Approach to Broadcasting in Multihop Radio Networks," IEEE Trans. Commun., vol. 39, pp. 426-433, Mar. 1991. 